\title{
Predictive Nomograms for Clinical Outcomes in Hepatitis B-Related Cirrhosis Patients Receiving Antiviral Therapy
}

\author{
Ran Cheng* \\ Jinghang $\mathrm{Xu}^{*}$ \\ Ning Tan \\ Hao Luo \\ Jiali Pan \\ Xiaoyuan Xu
}

Department of Infectious Diseases, Peking University First Hospital, Beijing,

People's Republic of China

*These authors contributed equally to this work.
Correspondence: Xiaoyuan Xu Department of Infectious Diseases, Peking University First Hospital, 8 Xishiku Street, Beijing, 100034, People's Republic of China

Tel/Fax +86-10-83575787

Email xiaoyuanxu6@163.com
Objective: Many scores have been constructed to predict liver-related events in chronic hepatitis B, while most of them are based on baseline clinical parameters. The objective of this study was to develop nomograms based on on-treatment improvement in established scores to predict clinical outcomes in patients with hepatitis B virus (HBV)-related cirrhosis who are receiving antiviral therapy.

Methods: The Cox proportional hazards regression model was used. Nomograms were constructed for the prediction of liver-related events, hepatocellular carcinoma (HCC), and liver-related mortality risk during long-term antiviral therapy.

Results: A total of 277 treatment-naive patients with $\mathrm{HBV}$-associated cirrhosis were enrolled from January 2010 to December 2013. After a median follow-up of 63.3 months, 95 patients developed liver-related events, including 59 patients with liver-related death. Multivariate Cox analysis showed that the albumin-bilirubin score at year 1 was an independent predictor of liver-related events, liver-related mortality, and HCC. Age, decompensation, and delayed virological remission were independent factors for liver-related mortality. Age was also a risk factor for liver-related events. The concordance index values of eventnomogram, mortality-nomogram, and HCC-nomogram were 0.742 (95\% confidence interval [CI], 0.691 0.793), 0.799 (95\% CI, 0.748 0.850), and 0.613 (95\% CI, 0.540 0.686), respectively. The calibration plots showed an agreement between the predicted and observed incidences, which indicates good calibration of the model of event-nomogram and mortalitynomogram.

Conclusion: The nomograms achieved an optimal preoperative prediction of liver-related events, mortality, and HCC development in patients with HBV-related cirrhosis receiving antiviral therapy. These findings may help to identify high-risk patients for further optimal surveillance and intervention strategies.

Keywords: antiviral therapy, liver-related events, hepatocellular carcinoma, liver-related mortality, albumin-bilirubin

\section{Introduction}

Approximately $3.5 \%$ of the global population is chronically infected with hepatitis $\mathrm{B}$ virus (HBV). ${ }^{1}$ Chronic hepatitis $\mathrm{B}(\mathrm{CHB})$ is a major cause of cirrhosis and hepatocellular carcinoma (HCC) in China. ${ }^{2,3}$ Long-term antiviral treatment with nucleos(t)ide analogs (NAs) can reduce necro-inflammatory activity, alleviate hepatic fibrosis or even reverse disease progression, and significantly reduce the incidence of liver-related events, including decompensation, HCC, and liver-related 
death. ${ }^{4-6}$ However, the risk of liver-related events cannot be eliminated in $\mathrm{CHB}$ patients undergoing NA therapy, particularly in those with pre-existing cirrhosis, as current antiviral therapy cannot eradicate the virus. ${ }^{7}$ HCC develops even in patients with hepatitis B surface antigen (HBsAg) seroclearance. ${ }^{8}$ Therefore, it is crucial to identify patients with cirrhosis who are at a high risk of developing liver-related events during long-term NA therapy.

Previous studies have constructed many prognostic models for the prediction of $\mathrm{HCC} ; ;^{9-12}$ however, few of them have been widely used in clinical practice due to differences in selected population characteristics. Furthermore, owing to their components, such as HBV DNA and alanine amino transferase (ALT), some of these models are less informative for predicting $\mathrm{HCC}$ in patients on long-term antiviral therapy than in untreated patients. In addition, the surveillance strategy and treatment differ between HCC and other causes of liver-related death. ${ }^{13}$ Recently, a small series of research studies indicated that on-treatment noninvasive indices, including liver stiffness measurement (LSM), ${ }^{14}$ fibrosis-4 (FIB$4),{ }^{15}$ and model for end-stage liver disease (MELD), ${ }^{16}$ or the albumin-bilirubin (ALBI) score, which was initially developed for assessing the severity of liver dysfunction in patients with $\mathrm{HCC},{ }^{17}$ showed great prognostic value for liver-related events. ${ }^{18}$

Therefore, the aim of this study was to investigate the predictors of developing liver-related events, HCC, and liver-related mortality in patients with CHB-related cirrhosis undergoing long-term NA therapy. Nomograms based on these independent risk predictors were then constructed to support clinicians in their treatment recommendations.

\section{Patients and Methods}

\section{Patients}

Treatment-naive patients with HBV-associated cirrhosis admitted to Peking University First Hospital between January 2010 and December 2013 were enrolled in this retrospective study. The inclusion criteria were as follows: (1) age between 18 and 75 years and (2) the start of NA treatment, without interruption, during follow-up. Eligible patients chose one of the following as their initial treatment: entecavir (ETV), lamivudine (LAM) plus adefovir (ADV), ADV monotherapy, LAM monotherapy, or telbivudine (LdT). The exclusion criteria were (1) other causes of chronic liver disease, such as hepatitis $\mathrm{C}$ virus, alcoholic liver disease, drug-induced liver injury, autoimmune liver disease, severe non-alcoholic fatty liver, or genetic liver disease; (2) combination of HCC or other malignancies; (3) other severe diseases, such as heart failure or chronic obstructive pulmonary disease; (4) previous liver transplantation; (5) death within 1 year; (6) incomplete laboratory parameters; and (7) co-infection with hepatitis $\mathrm{D}$ virus.

The protocol used in this study was approved by the ethics committee board of Peking University First Hospital, Beijing, China (Approval No. 2020363) and complied with the Declaration of Helsinki 1975. In addition, written informed consent from the patients or their relatives to participate in the study was obtained, and data were analyzed anonymously.

\section{Data Collection}

Clinical examinations, blood tests for biochemistry (including ALT, albumin [ALB], aspartate aminotransferase [AST], total bilirubin [TBIL], and serum creatinine [Scr]), the international normalized ratio (INR), blood platelet (PLT), and virologic parameters (HBsAg, hepatitis $\mathrm{B}$ e antigen [HBeAg], and HBV-DNA) were collected within 24 hours of admission and before antiviral treatment. After the initiation of antiviral therapy, follow-up observations of patients were continued for 6 years, including blood cell count, liver function tests, virologic parameters, and INR, which were conducted every 6 months until the last follow-up date. The incidence of all clinically relevant liver-related events was monitored through self-reports by the patients or by reviewing the clinical, laboratory, and imaging findings. Prognostic scores, including aspartate aminotransferase-to-platelet ratio index (APRI) ${ }^{19}$ FIB-4, ${ }^{20}$ ALBI score, ${ }^{17}$ and MELD score, ${ }^{21}$ were calculated using the formulas from the corresponding original articles.

\section{Definitions}

Liver cirrhosis was confirmed by: (1) liver biopsy, (2) medical imaging techniques indicating imaging changes in liver morphology, and (3) HBV infection with esophageal varices indicated by endoscopy or splenomegaly on radiologic images. Liver-related events were defined as HCC and liver-related death in our study. Virologic response (VR) was defined as serum HBV-DNA below the lower limit of detection. Virologic breakthrough (VB) was defined as an increase of $\geq 1 \log _{10} \mathrm{IU} / \mathrm{mL}$ compared with the nadir during treatment in patients with good adherence. For patients who did not receive ETV initially, 
the rescue therapy was ETV treatment. For those administered ETV initially, the rescue therapy was the addition of ADV $10 \mathrm{mg} /$ day or tenofovir (TDF) $300 \mathrm{mg} /$ day.

\section{Statistical Analysis}

Data were analyzed using SPSS for Windows (version 22.0, IBM, Armonk, NY, USA) and R software for Windows (version 4.0.3, http://www.r-project.org). A $P$-value $<0.05$ (two-tailed) was considered to denote a significant difference. Continuous variables are expressed as mean \pm standard deviation or median (interquartile range), whereas categorical variables are presented as numbers (percentages). Student's $t$-tests or non-parametric tests were used for continuous variables, and $\chi^{2}$ tests were used for categorical variables. Cumulative rates of liver-related events, $\mathrm{HCC}$, and liver-related mortality were calculated using Kaplan-Meier analysis. Liver-related event data were recorded when either of the following occurred first: HCC or death. Possible prognostic factors were subjected to univariate Cox proportional hazards regression analysis. Variables with $P$-values $<0.05$ were included in the multivariate analysis. The independent prognostic variables on multivariate analysis were selected to construct nomograms for assessing risk. The $\mathrm{C}$-index was calculated to evaluate the discrimination ability of the nomograms, whereas calibration plots were used to graphically evaluate the calibration of the nomograms. Area under receiver operating characteristic (AUROC) curve was used to predict HCC development. Comparisons of AUROC were performed using the method of DeLong et al. ${ }^{22}$

\section{Results}

\section{Patient Characteristics}

We identified 563 treatment-naive patients with HBVrelated cirrhosis who were treated with NAs. After the inclusion and exclusion criteria were considered, 277 patients were finally included in the analysis (Figure 1), including 112 patients with decompensated cirrhosis. The baseline characteristics of the study population are shown in Table 1. Their mean age was $52.41 \pm 10.22$ years; 211 (76.1\%) patients were male; 97 (35\%) patients were HBeAg-positive and 120 (43.3\%) patients received ETV.

After a median follow-up period of 63.3 months, 95 patients developed liver-related events; among them were 51 patients with HCC and 59 patients with liver-related death (including 15 patients who developed HCC before liver-related death). The 6-year cumulative incidence rates of liver-related events, liver-related mortality, and HCC were $34.3 \%, 21.3 \%$, and $18.4 \%$, respectively (Supplementary Figure 1A- - ).

As a baseline, patients with liver-related events were more likely to be older than patients without liver-related events; have lower ALB, ALT, and HBV-DNA; and have higher ALBI scores. Sex, BMI, HBeAg status, PLT, AST, TBIL, Scr, INR, FIB-4, APRI, and MELD were similar in the two groups (Table 1).

\section{Dynamic Changes in Non-Invasive Indicators During Treatment}

After the first year of antiviral treatment, 206 (74.3\%) patients achieved VR. A total of 27 (9.7\%) patients had rescue therapy due to VB. Among 182 patients without liver-related events, non-invasive indicators, including FIB-4, APRI, MELD, and ALBI, showed a rapid decrease from the baseline within 1 year after treatment and, continued to decline steadily but significantly from year 1 to year 6 after treatment (Supplementary Figure 2A-D). In contrast, for patients with liver-related events, there was a slightly increasing trend of the non-invasive indicators within the first year, with no significant change through the following 5 years (Supplementary Figure 3A-D).

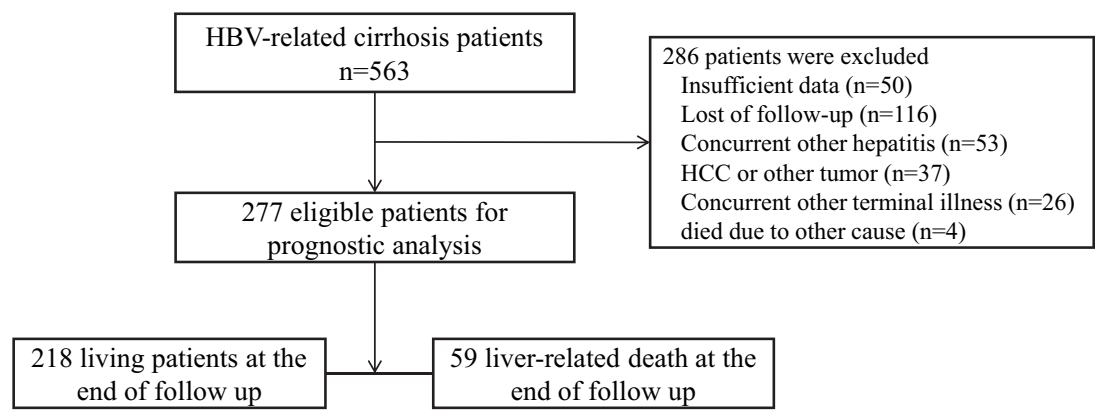

Figure I Flow chart of patients with hepatitis B virus-related cirrhosis. 
Table I Baseline Characteristics of Patients with HBV-Related Cirrhosis

\begin{tabular}{|c|c|c|c|c|}
\hline Parameters & Total $(n=277)$ & Without LREs $(n=182)$ & With LREs (n=95) & $P$-value \\
\hline Age (year) & $52.41 \pm 10.22$ & $51.13 \pm 10.02$ & $54.86 \pm 10.20$ & $0.005^{*}$ \\
\hline Male, n (\%) & $211(76.1 \%)$ & 140 (76.9\%) & $7 \mathrm{I}(74.7 \%)$ & 0.685 \\
\hline Follow-up (month) & $63.3 \pm 19.02$ & $72 \pm 0$ & $46.64 \pm 25.2$ & $<0.00 I^{*}$ \\
\hline BMI $\left(\mathrm{kg} / \mathrm{m}^{2}\right)$ & $24.72 \pm 3.23$ & $24.49 \pm 3.20$ & $25.14 \pm 3.26$ & 0.130 \\
\hline HBV DNA $\left(\log _{10} \mathrm{IU} / \mathrm{mL}\right)$ & $4.51(3.116 .1)$ & $4.91(3.126 .14)$ & $3.94(3.115 .75)$ & $0.033 *$ \\
\hline $\mathrm{HBeAg}(+), \mathrm{n}(\%)$ & 97 (35\%) & 60 (33\%) & 37 (38.9\%) & 0.322 \\
\hline PLT, $\times 10^{9} / \mathrm{L}$ & $79(56$ । I 8$)$ & $81(56 \mid 22)$ & $75(54 \quad 105)$ & 0.491 \\
\hline ALT, IU/L & $37(2568)$ & $38.5(2790.65)$ & $34(2355)$ & $0.026 *$ \\
\hline AST, IU/L & $46(3 \mid 76.5)$ & $46(3278.25)$ & $44(3 \mid 75)$ & 0.531 \\
\hline $\mathrm{Scr}, \mu \mathrm{mol} / \mathrm{L}$ & $78(6888)$ & 79 (69.93 88) & $76(6588)$ & 0.144 \\
\hline TBIL, $\mu \mathrm{mol} / \mathrm{L}$ & $22.8(14.138 .1)$ & $22.9(14.639 .3)$ & $21.6(12.835 .7)$ & 0.440 \\
\hline ALB, $g / L$ & $34.7(29.3540 .1)$ & $35.4(30.384 \mathrm{I} .5)$ & $33.4(2837.9)$ & $0.009 *$ \\
\hline INR & $1.19(1.081 .31)$ & 1.17 (1.08 I.29) & I.2I (1.08 I.32) & 0.212 \\
\hline Decompensation, n (\%) & $112(40.4 \%)$ & $55(30.2 \%)$ & $57(60 \%)$ & $<0.00 I^{*}$ \\
\hline VR at year I, n (\%) & $206(74.3 \%)$ & 145 (79.7\%) & $61(64.2 \%)$ & $0.005^{*}$ \\
\hline VB, n (\%) & $27(9.7 \%)$ & $13(7.1 \%)$ & $14(14.7 \%)$ & $0.043^{*}$ \\
\hline ETV treatment, n (\%) & $120(43.3 \%)$ & $82(37.5 \%)$ & 38 (40\%) & 0.420 \\
\hline FIB-4 & $5.4(3.218 .64)$ & 5.05 (3.07 8.39) & 5.87 (3.42 9.5) & 0.054 \\
\hline APRI & 1.71 (0.89 3.13) & 1.65 (0.89 3.08) & $1.74(0.83 .23)$ & 0.705 \\
\hline MELD & $10.06(8.16 \quad 12.3)$ & $9.75(8.1 \mid 12.3)$ & $10.4 \mid(8.16$ |2.49) & 0.410 \\
\hline ALBI & $-2.09(-2.55-1.52)$ & $-2.14(-2.69-1.64)$ & $-2.03(-2.45-1.34)$ & $0.032 *$ \\
\hline
\end{tabular}

Note: $* P<0.05$.

Abbreviations: ALB, albumin; ALBI, Albumin-bilirubin score; ALT, alanine amino transferase; APRI, Aspartate aminotransferase-to-platelet ratio index; AST, aspartate aminotransferase; BMI, body mass index; FIB-4, Fibrosis-4 score; HCC, hepatocellular carcinoma; HBeAg, hepatitis B e antigen; HBV, hepatitis B virus; INR, international normalized ratio; MELD, Model for end-stage liver disease score; PLT, blood platelet; TBIL, total bilirubin; VB, virologic breakthrough; VR, virologic response.

\section{Clinical Factors Associated with Liver-Related Events}

Liver-related events occurred in 95 of the 277 patients during the treatment period. Data regarding clinical and laboratory findings were considered potential prognostic factors affecting liver-related events and were included in Cox regression analysis. The results showed that decompensation, age, baseline HBV DNA, baseline ALB, baseline ALBI, HBV DNA positivity, FIB-4, APRI, MELD, and ALBI at year 1 of the treatment were associated with liver-related events (Table 2). To avoid redundancy, the individual components of the ALBI score significantly associated with mortality in the univariate analysis were not tested in the multivariate regression analysis. On this basis, the Cox multivariate stepwise regression analysis identified age and ALBI at year 1 of the treatment as independent indicators for the risk of liver-related events (Table 2).

Based on the independent factors demonstrated in the multivariate analysis, a nomogram was developed to predict 6-year liver-related events (Figure 2A). This model exhibited better efficacy in estimating liver-related events with a higher C-index of 0.742 (95\% confidence interval [CI] 0.691 0.793) than that of the ALBI score alone of 0.708 at year $1(95 \%$ CI $0.659 \sim 0.757)$. The calibration plots illustrated the predicted and observed incidences of liver-related events. (Figure 2B).

For instance, one patient with an age of 60 years old and ALBI $>-2.6$ at year 1 would have a predictive prognostic probability of at least $45 \%$, while for a patient with an age of 60 years old and ALBI $>-1.39$, the predictive probability would increase to approximately $85 \%$ (Figure 2A).

\section{Incidence and Predictors of Developing Liver-Related Mortality}

Of the 277 patients, 59 developed liver-related mortality during the treatment period. Data regarding clinical and laboratory findings were considered potential prognostic factors affecting liver-related death and were included in the Cox regression analysis. Table 3 shows the univariate analysis results of the baseline and on-treatment factors associated with liver-related mortality. The Cox multivariate stepwise regression analysis identified age, 
Table 2 Univariate and Multivariate Analyses of Predictive Factors Associated with Liver-Related Events

\begin{tabular}{|c|c|c|c|c|}
\hline \multirow[t]{2}{*}{ Parameters } & \multicolumn{2}{|c|}{ Univariate Analysis } & \multicolumn{2}{|c|}{ Multivariate Analyses } \\
\hline & HR (95\% Cl) & $P$ value & HR (95\% Cl) & $P$ value \\
\hline Age (year) & $1.033(1.013 \quad 1.053)$ & $0.00 I^{*}$ & 1.028 (1.009 1.049) & $0.005 *$ \\
\hline Male, n (\%) & 0.928 (0.584 I.475) & 0.753 & & \\
\hline BMI $\left(\mathrm{kg} / \mathrm{m}^{2}\right)$ & $1.046(0.984$ I.III) & 0.146 & & \\
\hline HBV DNA $\left(\log _{10} I U / m L\right)$ & $0.854(0.7530 .968)$ & 0.014 & & \\
\hline $\mathrm{HBeAg}(+), \mathrm{n}(\%)$ & $1.220(0.808 \quad 1.843)$ & 0.344 & & \\
\hline PLT, $\times 10^{9} / \mathrm{L}$ & $1.000(0.996 \quad$ I.004) & 0.966 & & \\
\hline ALT, IU/L & $0.997(0.995$ I.000) & 0.060 & & \\
\hline AST, IU/L & 0.999 (0.997 I.00I) & 0.255 & & \\
\hline $\mathrm{Scr}, \mu \mathrm{mol} / \mathrm{L}$ & $0.996(0.987 \quad 1.005)$ & 0.421 & & \\
\hline TBIL, $\mu \mathrm{mol} / \mathrm{L}$ & $1.003(0.998 \quad 1.009)$ & 0.236 & & \\
\hline ALB, $g / L$ & $0.963(0.9360 .991)$ & $0.009 *$ & & \\
\hline INR & $1.625(0.95 \mid 2.776)$ & 0.076 & & \\
\hline ETV treatment, $\mathrm{n}(\%)$ & $0.864(0.573 \quad$ I.303 $)$ & 0.487 & & \\
\hline Decompensation, n (\%) & 2.786 (1.846 4.205) & $0.000 *$ & & \\
\hline FIB-4 at baseline & 1.015 (0.990 I.042) & 0.237 & & \\
\hline APRI at baseline & $0.99 \mid(0.96|\quad| .022)$ & 0.567 & & \\
\hline \multicolumn{5}{|l|}{ MELD at baseline } \\
\hline \multicolumn{5}{|l|}{ MELD $(\leq 10)$} \\
\hline MELD $(10 \sim 15)$ & $1.448(0.94 \mid 2.226)$ & 0.092 & & \\
\hline MELD (>15) & I. 102 (0.579 2.097) & 0.767 & & \\
\hline \multicolumn{5}{|l|}{ ALBI at baseline } \\
\hline \multicolumn{5}{|l|}{ ALBI $(\leq-2.6)$} \\
\hline ALBI $(-2.6 \sim-1.39)$ & 1.574 (0.887 2.793) & 0.121 & & \\
\hline ALBI (>-1.39) & $2.117(1.1263 .98 I)$ & 0.020 & & \\
\hline Virologic breakthrough, n (\%) & $1.533(0.8692 .705)$ & 0.140 & & \\
\hline HBV-DNA positivity at year I of the treatment & $1.790(1.1762 .724)$ & $0.007^{*}$ & & \\
\hline FIB-4 at year I of the treatment & $1.028\left(\begin{array}{lll}1.020 & 1.036\end{array}\right)$ & $<0.00 I^{*}$ & & \\
\hline APRI at year I of the treatment & $\mathrm{I} .047$ (I.032 I.062) & $<0.00 I^{*}$ & & \\
\hline \multicolumn{5}{|l|}{ MELD at year I of the treatment } \\
\hline \multicolumn{5}{|l|}{ MELD $(\leq 10)$} \\
\hline MELD $(10 \sim 15)$ & $1.965(1.2293 .140)$ & $0.005^{*}$ & & \\
\hline MELD (>15) & $8.107(4.797$ I3.70I) & $<0.00 I^{*}$ & & \\
\hline \multicolumn{5}{|l|}{ ALBI at year I of the treatment } \\
\hline \multicolumn{5}{|l|}{ ALBI $(\leq-2.6)$} \\
\hline ALBI $(-2.6 \sim-1.39)$ & 2.208 (1.299 3.754) & $0.003 *$ & $1.463(0.8102 .643)$ & 0.207 \\
\hline ALBI (>-I.39) & $9.744(5.619$ 16.896) & $<0.00 I^{*}$ & $3.143(1.4476 .825)$ & $0.004^{*}$ \\
\hline
\end{tabular}

Note: $* P<0.05$.

Abbreviations: ALB, albumin; ALBI, Albumin-bilirubin score; ALT, alanine amino transferase; APRI, Aspartate aminotransferase-to-platelet ratio index; AST, aspartate aminotransferase; BMI, body mass index; FIB-4, Fibrosis-4 score; HCC, hepatocellular carcinoma; $\mathrm{HBeAg}$, hepatitis B e antigen; HBV, hepatitis B virus; INR, international normalized ratio; MELD, Model for end-stage liver disease score; PLT, blood platelet; TBIL, total bilirubin; VB, virologic breakthrough; VR, virologic response.

decompensation, HBV-DNA positivity, and ALBI at year 1 of the treatment as independent indicators for the risk of liver-related death (Table 3).

Based on the independent factors demonstrated in the multivariate analysis, a nomogram was developed to predict 6-year liver-related mortality (Figure 3A). This model exhibited better efficacy in estimating mortality with a higher C-index of 0.799 (95\% CI $0.748 \sim 0.850$ ) than that of the ALBI score of 0.75 at year 1 alone (95\% CI 0.691 0.809). The calibration plots illustrated the predicted and observed incidences of liver-related mortality (Figure 3B). 


\section{A}

Points

$\begin{array}{llllllllll}0 & 10 & 20 & 30 & 40 & 50 & 60 & 70 & 80 & 90\end{array}$

Age

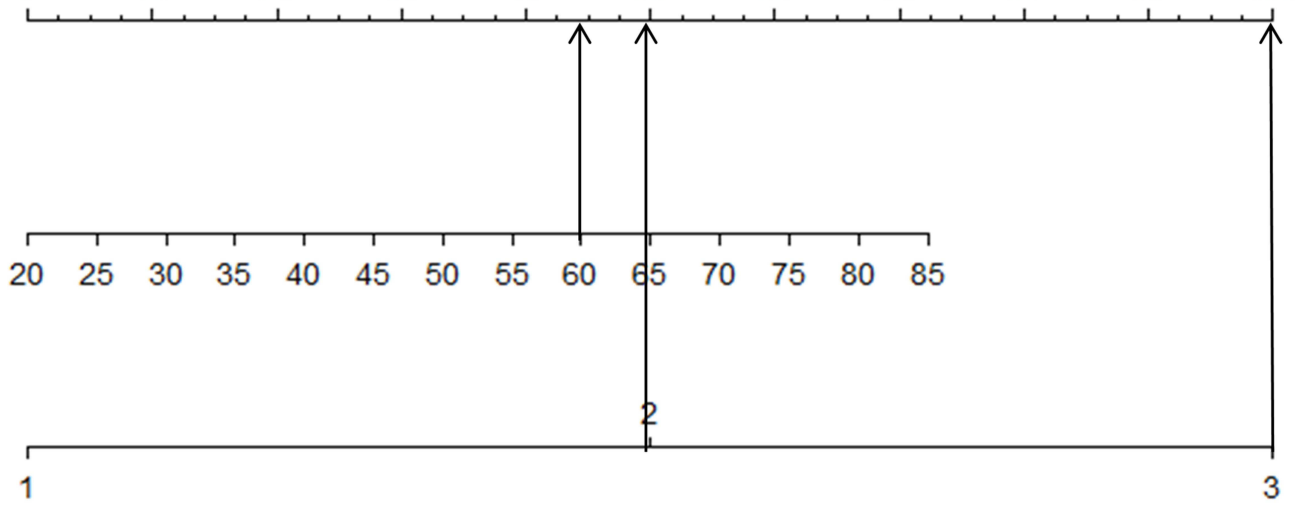

ALBI at year 1

Total Points

\begin{tabular}{llll|lll|lll}
\hline 0 & 20 & 40 & 60 & 80 & 100 & 120 & 140 & 160 & 180
\end{tabular}

Risk of liver-related events

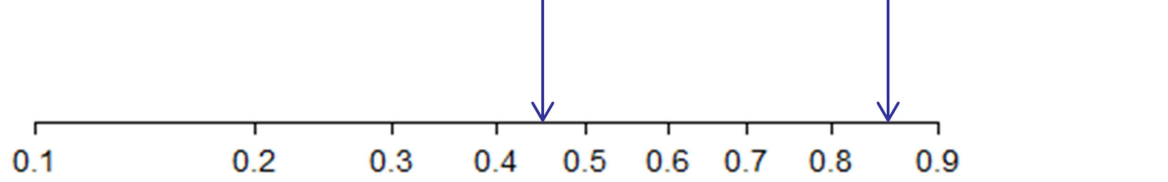

B

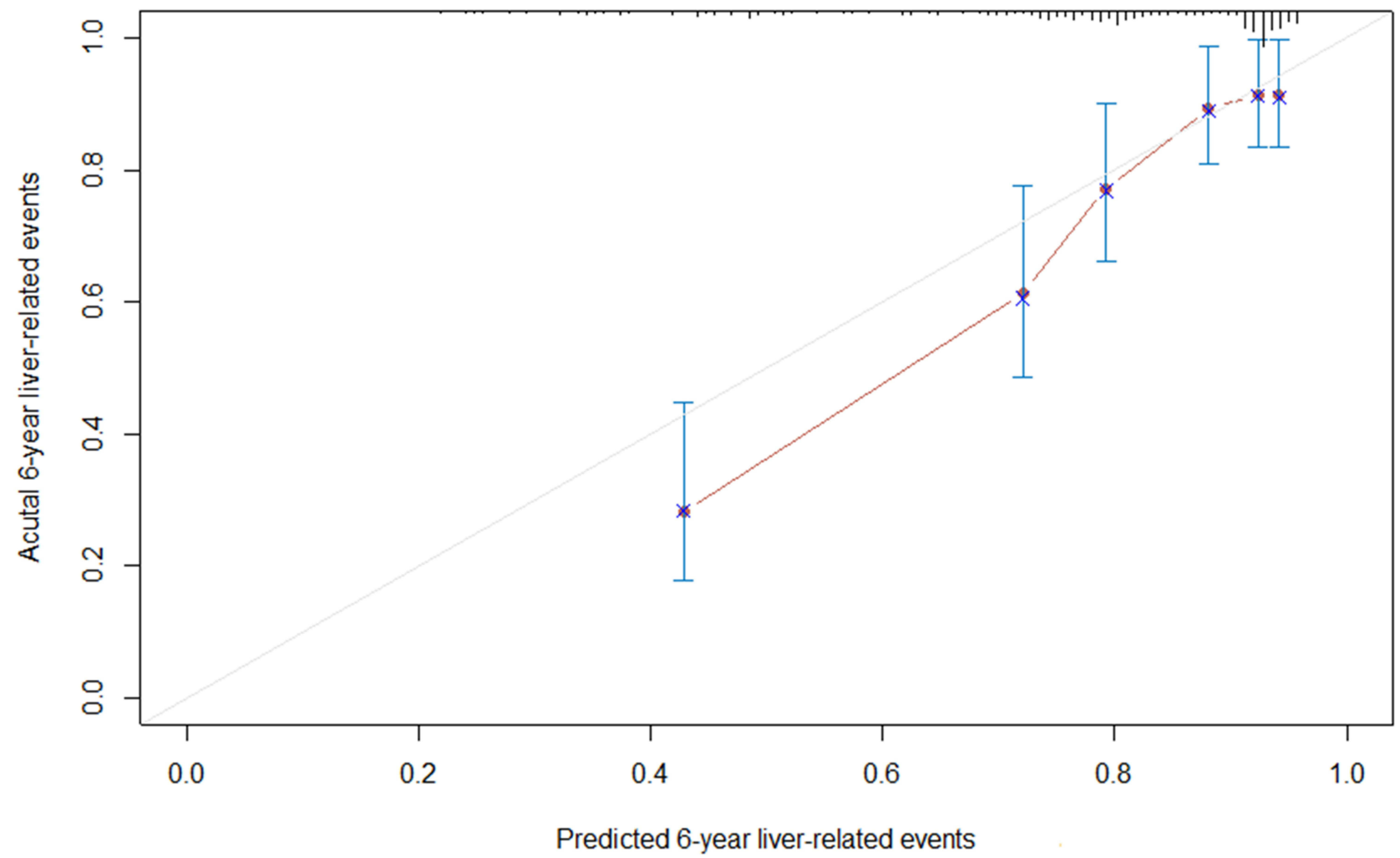

Figure 2 Nomogram predicting the development of liver-related events (A). Calibration plot for predicting liver-related events (B). 
Table 3 Univariate and Multivariate Analyses of Predictive Factors Associated with Liver-Related Mortality

\begin{tabular}{|c|c|c|c|c|}
\hline \multirow[t]{2}{*}{ Parameters } & \multicolumn{2}{|c|}{ Univariate Analysis } & \multicolumn{2}{|c|}{ Multivariate Analyses } \\
\hline & HR (95\% Cl) & $P$ value & HR $(95 \% \mathrm{Cl})$ & $P$ value \\
\hline Age (year) & $1.037(1.0111 .063)$ & $0.005 *$ & $\mathrm{I} .028(\mathrm{I} .004 \mathrm{I} .05 \mathrm{I})$ & $0.020 *$ \\
\hline Male, n (\%) & $1.020(0.560 \quad$ I.858) & 0.949 & & \\
\hline BMI $\left(\mathrm{kg} / \mathrm{m}^{2}\right)$ & $1.032(0.956$ I.II3) & 0.421 & & \\
\hline HBV DNA $\left(\log _{10} \mathrm{IU} / \mathrm{mL}\right)$ & $0.842(0.7170 .990)$ & $0.037^{*}$ & & \\
\hline $\mathrm{HBeAg}(+), \mathrm{n}(\%)$ & 1.056 (0.620 I.799) & 0.842 & & \\
\hline $\mathrm{PLT}, \times 10^{9} / \mathrm{L}$ & 1.004 (1.000 I.007) & 0.065 & & \\
\hline ALT, IU/L & $0.997(0.992 \quad 1.001)$ & 0.111 & & \\
\hline AST, IU/L & 0.998 (0.995 I.00I) & 0.251 & & \\
\hline $\mathrm{Scr}, \mu \mathrm{mol} / \mathrm{L}$ & 0.996 (0.987 I.005) & 0.421 & & \\
\hline TBIL, $\mu \mathrm{mol} / \mathrm{L}$ & $1.006(1.0001 .012)$ & $0.048^{*}$ & & \\
\hline ALB, $g / L$ & $0.942(0.9080 .977)$ & $0.00 I^{*}$ & & \\
\hline INR & $0.987(0.97 \mid \quad 1.003)$ & 0.114 & & \\
\hline ETV treatment, n (\%) & $\mathrm{I} .046$ (0.625 I.748) & 0.865 & & \\
\hline Decompensation, n (\%) & $3.410(1.9885 .850)$ & $<0.001^{*}$ & $2.018(1.1143 .657)$ & $0.021^{*}$ \\
\hline FIB-4 at baseline & I.007 (0.970 I.045) & 0.710 & & \\
\hline APRI at baseline & 0.990 (0.949 I.033) & 0.635 & & \\
\hline \multicolumn{5}{|l|}{ MELD at baseline } \\
\hline \multicolumn{5}{|l|}{$\operatorname{MELD}(\leq 10)$} \\
\hline MELD $(10 \sim 15)$ & 1.577 (0.910 2.734) & 0.104 & & \\
\hline MELD $(>15)$ & $1.296(0.5822 .884)$ & 0.526 & & \\
\hline \multicolumn{5}{|l|}{ ALBI at baseline } \\
\hline \multicolumn{5}{|l|}{$\mathrm{ALBI}(\leq-2.6)$} \\
\hline ALBI $(-2.6 \sim-1.39)$ & $2.590(1.0876 .169)$ & 0.032 & & \\
\hline ALBI (>-1.39) & $3.777(1.5089 .460)$ & 0.005 & & \\
\hline VB, n (\%) & 1.380 (0.655 2.909) & 0.397 & & \\
\hline HBV-DNA positivity at year I of the treatment & $2.497(1.4934 .178)$ & $<0.00 I^{*}$ & $1.857(1.0943 .155)$ & $0.022 *$ \\
\hline FIB-4 at year I of the treatment & $1.032\left(\begin{array}{lll}1.023 & 1.042)\end{array}\right)$ & $<0.00 I^{*}$ & & \\
\hline APRI at year I of the treatment & 1.029 (1.017 I.042) & $<0.00 I^{*}$ & & \\
\hline \multicolumn{5}{|l|}{ MELD at year I of the treatment } \\
\hline \multicolumn{5}{|l|}{$\operatorname{MELD}(\leq 10)$} \\
\hline MELD $(10 \sim 15)$ & $2.213(1.1504 .258)$ & $0.017^{*}$ & & \\
\hline MELD (>15) & $15.846(8.35830 .042)$ & $<0.00 I^{*}$ & & \\
\hline \multicolumn{5}{|l|}{$A L B I$ at year I of the treatment } \\
\hline \multicolumn{5}{|l|}{$\operatorname{ALBI}(\leq-2.6)$} \\
\hline ALBI $(-2.6 \sim-1.39)$ & $2.916(1.3496 .301)$ & $0.007^{*}$ & $1.899(0.8344 .325)$ & 0.127 \\
\hline ALBI (>-I.39) & $|4.02|$ (6.564 29.95) & $<0.00 I^{*}$ & 7.721 (3.309 18.017) & $0.000 *$ \\
\hline
\end{tabular}

Note: $* P<0.05$.

Abbreviations: ALB, albumin; ALBI, Albumin-bilirubin score; ALT, alanine amino transferase; APRI, Aspartate aminotransferase-to-platelet ratio index; AST, aspartate aminotransferase; BMI, body mass index; FIB-4, Fibrosis-4 score; HCC, hepatocellular carcinoma; $\mathrm{HBeAg}$, hepatitis B e antigen; HBV, hepatitis B virus; INR, international normalized ratio; MELD, Model for end-stage liver disease score; PLT, blood platelet; TBIL, total bilirubin; VB, virologic breakthrough; VR, virologic response.

In this nomogram, a decompensated patient with an age of 60 years old, HBV-DNA positivity and ALBI > 2.6 at year 1 would have a predictive prognostic probability of at least $50 \%$, while for a patient with an ALBI > -1.39 at year 1 , the predictive probability would increase to approximately $90 \%$ (Figure 3A).

\section{Incidence and Predictors of Developing HCC}

Of the 277 patients, 51 developed HCC during treatment. Data regarding clinical and laboratory findings were considered potential prognostic factors affecting $\mathrm{HCC}$ and were included in Cox regression analysis. In 
A

Points

Age

ALBI at year 1

decompensation

HBV DNA positivity at year 1

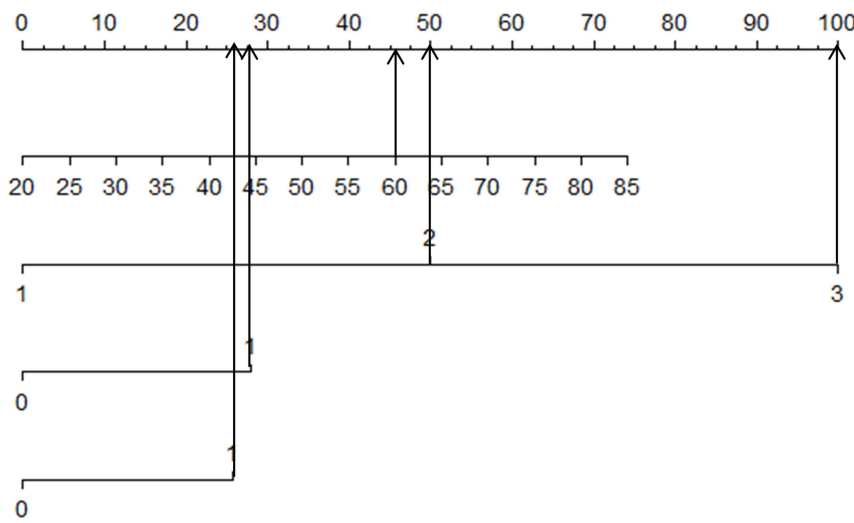

Total Points

Risk of liver-related death

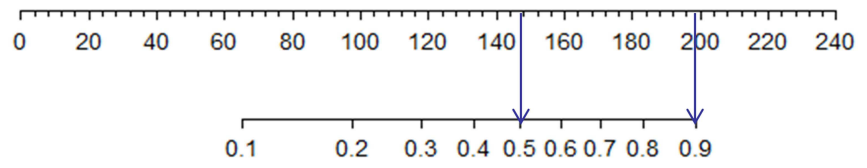

B

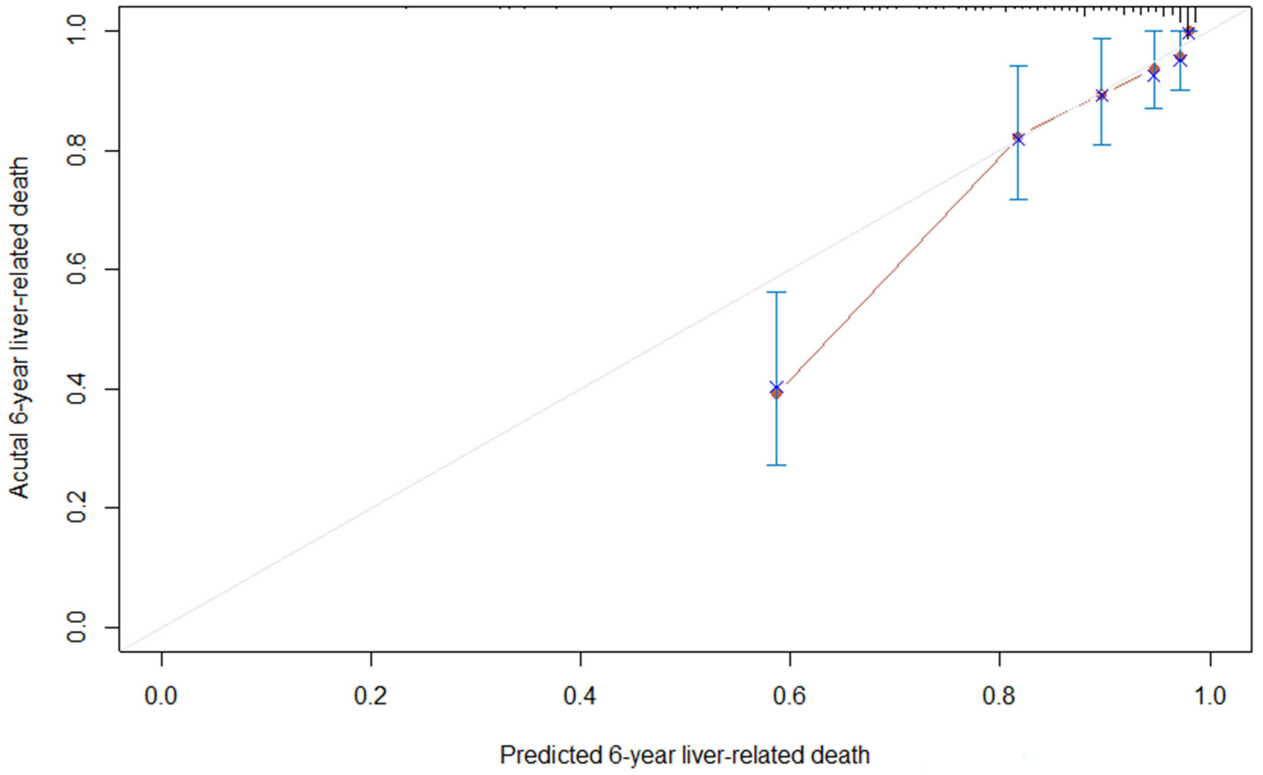

Figure 3 Nomogram predicting the development of liver-related mortality (A). Calibration plot for predicting liver-related mortality (B).

all the patients, APRI and ALBI at year 1 of the treatment showed significant associations with HCC according to univariate Cox regression analysis (Table 4). By employing multivariate stepwise regression analysis, we identified ALBI at year 1 of the treatment as an independent indicator for the risk of HCC (Table 4).

Based on the independent factors demonstrated in the multivariate analysis, a nomogram was developed to predict 6-year HCC (Figure 4A). This model exhibited efficacy in estimating mortality with a C-index of 0.613 (95\% CI 0.540 0.686). The calibration plots illustrated the predicted and observed incidences of HCC (Figure 4B). For predicting HCC, the model at year 1 had the best fit, though the AUROC was not significantly different compared with previous models of AASL-H, ${ }^{23}$ CU-HCC, ${ }^{24}$ mPAGE-B, ${ }^{25}$ and PAGE-B. ${ }^{9}$ (Supplementary Table 1 , Supplementary Figure 4) 
Table 4 Univariate and Multivariate Analyses of Predictive Factors Associated with HCC

\begin{tabular}{|c|c|c|c|c|}
\hline \multirow[t]{2}{*}{ Parameters } & \multicolumn{2}{|c|}{ Univariate Analysis } & \multicolumn{2}{|c|}{ Multivariate Analyses } \\
\hline & HR (95\% Cl) & $P$ value & HR (95\% CI) & $P$ value \\
\hline Age (year) & $\mathrm{I} .022(0.995 \mathrm{I} .050)$ & 0.108 & & \\
\hline Male, n (\%) & 0.658 (0.364 I.I89) & 0.166 & & \\
\hline BMI $\left(\mathrm{kg} / \mathrm{m}^{2}\right)$ & I.06I (0.977 I.I53) & 0.157 & & \\
\hline HBV DNA $\left(\log _{10} \mathrm{IU} / \mathrm{mL}\right)$ & $0.842(0.787$ I.100) & 0.400 & & \\
\hline $\mathrm{HBeAg}(+), \mathrm{n}(\%)$ & $1.419(0.8152 .469)$ & 0.216 & & \\
\hline PLT, $\times 10^{9} / \mathrm{L}$ & $1.004(0.987$ I.000) & 0.050 & & \\
\hline ALT, IU/L & $0.998(0.995$ I.00I) & 0.233 & & \\
\hline AST, IU/L & 0.999 (0.997 I.002) & 0.575 & & \\
\hline Scr, $\mu \mathrm{mol} / \mathrm{L}$ & 0.998 (0.990 I.006) & 0.635 & & \\
\hline TBIL, $\mu \mathrm{mol} / \mathrm{L}$ & 0.994 (0.983 I.005) & 0.274 & & \\
\hline ALB, $g / L$ & 0.997 (0.960 I.036) & 0.871 & & \\
\hline ETV treatment, n (\%) & 0.697 (0.392 ।.238) & 0.218 & & \\
\hline Decompensation, n (\%) & $1.610(0.9302 .788)$ & 0.089 & & \\
\hline FIB-4 at baseline & $1.023(0.99 \mid \quad 1.056)$ & 0.168 & & \\
\hline APRI at baseline & 0.99 I (0.949 I.036) & 0.698 & & \\
\hline \multicolumn{5}{|l|}{ MELD at baseline } \\
\hline \multicolumn{5}{|l|}{$\operatorname{MELD}(\leq 10)$} \\
\hline MELD $(10 \sim 15)$ & I.I59 (0.645 2.08I) & 0.622 & & \\
\hline MELD (>15) & $0.922(0.3772 .255)$ & 0.858 & & \\
\hline \multicolumn{5}{|l|}{ ALBI at baseline } \\
\hline \multicolumn{5}{|l|}{ ALBI $(\leq-2.6)$} \\
\hline ALBI $(-2.6 \sim-1.39)$ & $1.050(0.5232 .109)$ & 0.891 & & \\
\hline ALBI (>-I.39) & I.I06 (0.488 2.506) & 0.810 & & \\
\hline VB, n (\%) & $2.003(0.9754 .116)$ & 0.059 & & \\
\hline HBV-DNA positivity at year I of the treatment & $1.529(0.8542 .736)$ & 0.153 & & \\
\hline FIB-4 at year I of the treatment & 1.009 (0.992 I.027) & 0.309 & & \\
\hline APRI at year I of the treatment & 1.028 (1.007 I.049) & $0.010^{*}$ & & \\
\hline \multicolumn{5}{|l|}{ MELD at year I of the treatment } \\
\hline \multicolumn{5}{|l|}{ MELD $(\leq 10)$} \\
\hline MELD $(10 \sim 15)$ & $1.586(0.8922 .819)$ & 0.116 & & \\
\hline $\operatorname{MELD}(>15)$ & $0.807(0.2822 .313)$ & 0.690 & & \\
\hline \multicolumn{5}{|l|}{ ALBI at year I of the treatment } \\
\hline ALBI $(\leq-2.6)$ & & & & \\
\hline ALBI $(-2.6 \sim-1.39)$ & $1.920(0.9933 .713)$ & $0.052 *$ & $1.920(0.9933 .713)$ & 0.052 \\
\hline ALBI $(>-1.39)$ & $2.987(1.4046 .357)$ & $0.005^{*}$ & 2.987 ( 1.404 6.357) & $0.005^{*}$ \\
\hline
\end{tabular}

Note: $* P<0.05$.

Abbreviations: ALB, albumin; ALBI, Albumin-bilirubin score; ALT, alanine amino transferase; APRI, Aspartate aminotransferase-to-platelet ratio index; AST, aspartate aminotransferase; BMI, body mass index; FIB-4, Fibrosis-4 score; HCC, hepatocellular carcinoma; HBeAg, hepatitis B e antigen; HBV, hepatitis B virus; INR, international normalized ratio; MELD, Model for end-stage liver disease score; PLT, blood platelet; TBIL, total bilirubin; VB, virologic breakthrough; VR, virologic response.

\section{Discussion}

In our study, the 6-year cumulative incidence of liverrelated events was lower than the reported rate in untreated patients. ${ }^{26}$ Nonetheless, $34.3 \%$ of patients with cirrhosis still developed liver-related events during 6-year antiviral treatment; $21.3 \%$ developed liver-related mortality alone; and $18.4 \%$ developed HCC alone in our study. Therefore, identifying risk determinants of HCC and mortality surveillance at early stages is indispensable for patients receiving long-term antiviral therapy. Previous studies have revealed determinants of liver-related events; however, the reported predictors have not been 
A

Points

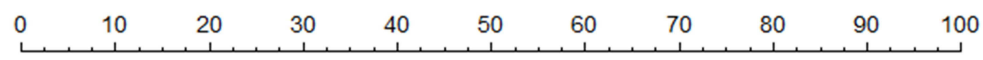

ALBI at year 1

2

1

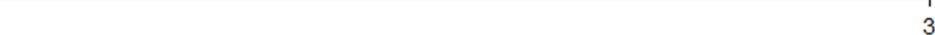

Total Points

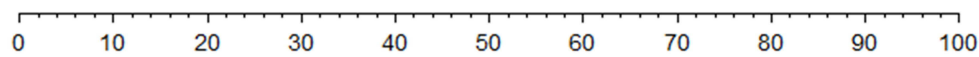

Risk of HCC

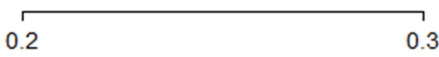

B

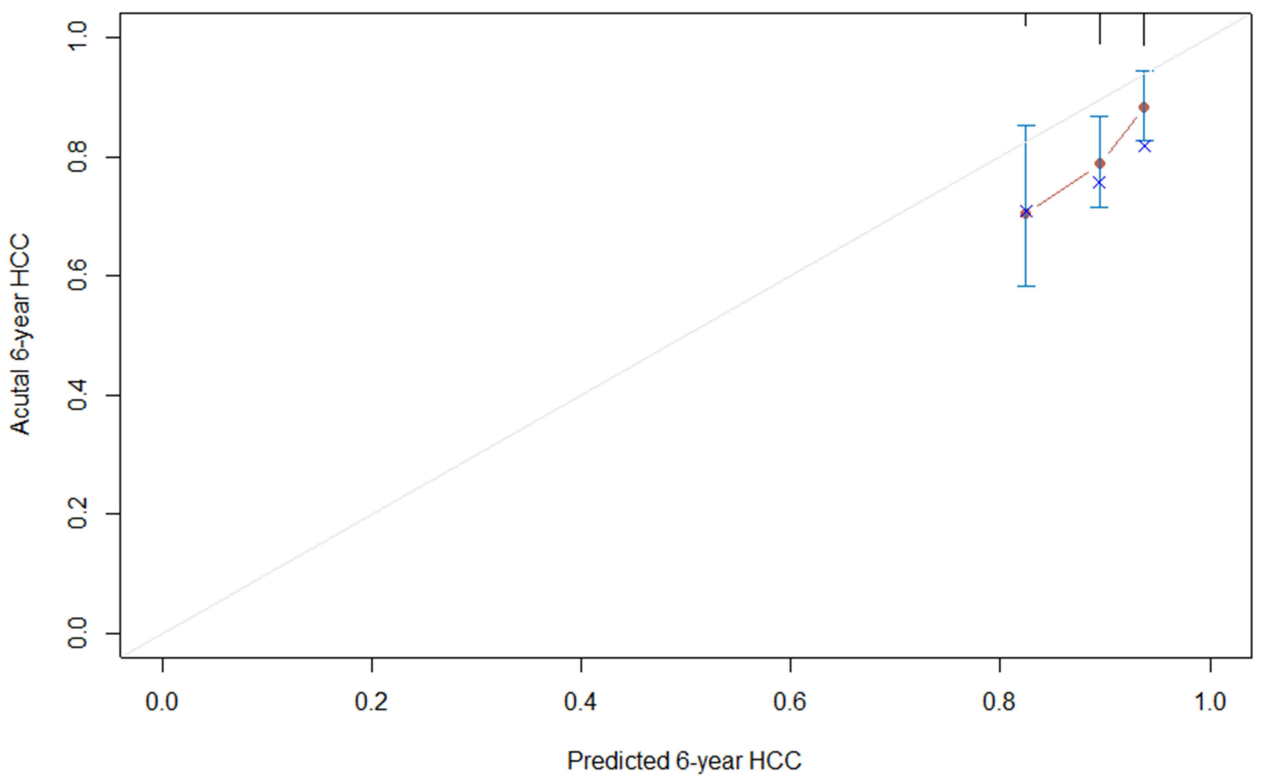

Figure 4 Nomogram predicting the development of hepatocellular carcinoma $(\mathrm{HCC})(\mathbf{A})$. Calibration plot for predicting $\mathrm{HCC}(\mathbf{B})$

consistent. $^{14,16,27}$ In this retrospective study, we determined independent risk predictors for liver-related events, liver-related mortality, and HCC from widely validated models developed with routinely available laboratory parameters.

The ALBI score is based on an algorithm combining two easily accessible and noninvasive blood test markers, while minimizing the need to include subjective factors (such as ascites and encephalopathy). ${ }^{17}$ Previous studies have shown that the ALBI score predicts long-term outcomes among patients with HBV-related cirrhosis. ${ }^{18,28}$ In our study, patients with a lower on-treatment ALBI score at year 1 had a lower risk of mortality and HCC development than that of patients with a higher ALBI score. The 
ALBI score at year 1 of treatment demonstrated better predictive performance for liver-related events, HCC development, and liver-related mortality than that at the baseline. We systematically explored the predictive performance of noninvasive indices and observed changes between the baseline and 1 year of treatment to determine the risk factors of liver-related events, $\mathrm{HCC}$, and mortality in patients with cirrhosis receiving long-term antiviral therapy. Finally, we demonstrated that the ALBI score at year 1 of antiviral treatment was a determinant for the development of liver-related events, $\mathrm{HCC}$, and mortality. Thus, on-treatment ALBI is a useful marker for predicting clinical outcomes in patients with cirrhosis undergoing antiviral therapy. On-treatment ALBI reflects improvements in biochemical and histological parameters and modification of the natural history of patients with HBVrelated cirrhosis. The results of this study confirm the efficacy of antiviral therapy and emphasize the importance of on-treatment surveillance of patients.

The primary aim of antiviral treatment is the complete suppression of viral replication. In our study, HBV-DNA positivity at year 1 of treatment was an independent predictor of liver-related mortality. The present investigation confirmed that a partial VR or no response is associated with an increased risk of liver-related mortality in patients with cirrhosis receiving antiviral therapy. A sustained VR is associated with improvements in liver histology and clinical outcomes, including decompensation, HCC development, and liver-related mortality. ${ }^{29-31}$ Delayed virologic remission is associated with an increased risk of HCC and fibrosis progression in patients with $\mathrm{CHB}$ during NA therapy. $^{32}$ Therefore, it is necessary to suppress HBV replication to an undetectable level as quickly as possible.

Besides the prediction of liver-related events, we also investigated predictive models for liver-related mortality and $\mathrm{HCC}$ alone. As demonstrated, independent predictors for the prediction of liver-related events, liver-related mortality, and HCC are different. Age is an independent predictor for both liver-related events and liver-related mortality but not for HCC. Decompensation and delayed virologic remission are independent predictors only for liver-related mortality.

In our research, age, sex, and HBV-DNA do not affect HCC. ALBI was the only independent predictor for HCC. The study systematically evaluated the predictive value, and illustrated that the ALBI score at year 1 of treatment is better than the previous HCC prediction models of AASL$\mathrm{H}, \mathrm{CU}-\mathrm{HCC}$, mPAGE-B, and PAGE-B. However, none of the prediction models had an excellent value, possibly due to the target population.

There were some limitations of our study. First, this was a single-center retrospective study. Cross validation and further investigations of these nomograms in a prospective multicenter study should be conducted in future research. Second, not all of the patients included in our study received first-line treatment options. Those who received low genetic barrier drugs might have had higher rates of suboptimal response or VB during antiviral treatment. Therefore, we are not certain whether drug resistance plays a role in liver-related events. Finally, the presence of cirrhosis was defined by physicians using different criteria (liver biopsy, LSM, or videography); thus bias cannot be eliminated.

\section{Conclusions}

Antiviral treatment decreases but does not eliminate the risk of $\mathrm{HCC}$ and liver-related mortality in patients with cirrhosis. Nomograms using on-treatment values are more accurate in predicting liver-related events, liver-related mortality, and HCC than are baseline values. Our study demonstrates that following 1 year of treatment, the ALBI score and age are useful markers for predicting liverrelated events in patients with cirrhosis receiving longterm antiviral therapy. Age, decompensation, and delayed VR are independent predictors for liver-related mortality but not for HCC development. Further prospective studies with larger sample sizes are needed to confirm these findings.

\section{Abbreviations}

ALB, albumin; ALBI, albumin-bilirubin; ALT, alanine amino transferase; APRI, aspartate aminotransferase to platelet ratio index; AST, aspartate aminotransferase; $\mathrm{BMI}$, body mass index; $\mathrm{CHB}$, chronic hepatitis $\mathrm{B}$; $\mathrm{CI}$, confidence interval; DNA, deoxyribonucleic acid; FIB-4, fibrosis index based on the four factors; HAV, hepatitis A virus; HBsAg, hepatitis B surface antigen; $\mathrm{HBeAg}$, hepatitis B e antigen; HBV, hepatitis B virus; HCC, hepatocellular carcinoma; $\mathrm{HCV}$, hepatitis $\mathrm{C}$ virus; HDV, hepatitis D virus; HR, hazard ratio; HIV, human immunodeficiency virus; INR, international normalized ratio; IQR, interquartile range; MELD, Model for EndStage Liver Disease; NA, Nucleos(t)ide analogs; PLT, blood platelet; Scr, serum creatinine; TBIL, total bilirubin; ULN, upper limit of normal; VR, Virologic response. 


\section{Acknowledgments}

We thank all the physicians and technicians whose work contributed to this study.

\section{Author Contributions}

All authors made substantial contribution to the work reported, whether that is in the conception, study design, acquisition of data, or analysis and interpretation, took part in drafting, revising or critically reviewing the article; agreed to submit to the current journal, gave final approval of the version to be published; and agree to be accountable for all aspects of the work.

\section{Funding}

This work was supported by The National Science and Technology Major Project for Infectious Diseases (No. 2017ZX10302201-004-009, No. 2017ZX10203202003); Beijing Municipal Science and Technology Commission of Major Projects (No. D161100002716002, No. D161100002716003, No. D161100003117005).

\section{Disclosure}

The authors declare that they have no competing interests.

\section{References}

1. Yuen MF, Chen DS, Dusheiko GM, et al. Hepatitis B virus infection. Nat Rev Dis Primers. 2018;4:18035. doi:10.1038/nrdp.2018.35

2. Terrault NA, Bzowej NH, Chang KM, Hwang JP, Jonas MM, Murad MH. AASLD guidelines for treatment of chronic hepatitis B. Hepatology. 2016;63(1):261-283. doi:10.1002/hep.28156

3. Lok AS, McMahon BJ. Chronic hepatitis B: update 2009. Hepatology. 2009;50(3):661-662. doi:10.1002/hep.23190

4. Wang BQ, Wang YL, Shi KQ. Four-year entecavir therapy reduces hepatocellular carcinoma, cirrhotic events and mortality in chronic hepatitis B patients. Liver Int. 2017;37(2):309-310. doi:10.1111/ liv. 13289

5. Chang TT, Liaw YF, Wu SS, et al. Long-term entecavir therapy results in the reversal of fibrosis/cirrhosis and continued histological improvement in patients with chronic hepatitis B. Hepatology. 2010;52 (3):886-893. doi:10.1002/hep. 23785

6. Hosaka T, Suzuki F, Kobayashi M, et al. Long-term entecavir treatment reduces hepatocellular carcinoma incidence in patients with hepatitis B virus infection. Hepatology. 2013;58(1):98-107. doi: $10.1002 /$ hep. 26180

7. Yang SC, Lee CM, Hu TH, et al. Virological response to entecavir reduces the risk of liver disease progression in nucleos $(\mathrm{t})$ ide analogue-experienced HBV-infected patients with prior resistant mutants. J Antimicrob Chemother. 2013;68(9):2154-2163. doi:10.1093/jac/dkt147

8. Yip TC, Wong GL, Chan HL, et al. HBsAg seroclearance further reduces hepatocellular carcinoma risk after complete viral suppression with nucleos(t)ide analogues. $J$ Hepatol. 2019;70(3):361-370. doi:10.1016/j.jhep.2018.10.014

9. Papatheodoridis G, Dalekos G, Sypsa V, et al. PAGE-B predicts the risk of developing hepatocellular carcinoma in Caucasians with chronic hepatitis B on 5-year antiviral therapy. J Hepatol. 2016;64 (4):800-806. doi:10.1016/j.jhep.2015.11.035
10. Poh Z, Shen L, Yang HI, et al. Real-world risk score for hepatocellular carcinoma (RWS-HCC): a clinically practical risk predictor for HCC in chronic hepatitis B. Gut. 2016;65(5):887-888. doi:10.1136/ gutjnl-2015-310818

11. Yang HI, Sherman M, Su J, et al. Nomograms for risk of hepatocellular carcinoma in patients with chronic hepatitis B virus infection. J Clin Oncol. 2010;28(14):2437-2444. doi:10.1200/JCO.2009.27.4456

12. Hsu YC, Yip TC, Ho HJ, et al. Development of a scoring system to predict hepatocellular carcinoma in Asians on antivirals for chronic hepatitis B. J Hepatol. 2018;69(2):278-285. doi:10.1016/j.jhep.2018.02.032

13. European Association for The Study of the Liver. EASL Clinical Practice Guidelines for the management of patients with decompensated cirrhosis. J Hepatol. 2018;69(2):406-460.

14. Wu S, Kong Y, Piao H, et al. On-treatment changes of liver stiffness at week 26 could predict 2-year clinical outcomes in HBV-related compensated cirrhosis. Liver Int. 2018;38(6):1045-1054. doi:10.1111/liv.13623

15. Chiang $\mathrm{HH}$, Lee $\mathrm{CM}, \mathrm{Hu} \mathrm{TH}$, et al. A combination of the on-treatment FIB-4 and alpha-foetoprotein predicts clinical outcomes in cirrhotic patients receiving entecavir. Liver Int. 2018;38 (11):1997-2005. doi:10.1111/liv.13889

16. Yip TC, Chan HL, Tse YK, et al. On-Treatment Improvement of MELD score reduces death and hepatic events in patients with hepatitis B-related cirrhosis. Am J Gastroenterol. 2018;113 (11):1629-1638. doi:10.1038/s41395-018-0247-9

17. Johnson PJ, Berhane S, Kagebayashi C, et al. Assessment of liver function in patients with hepatocellular carcinoma: a new evidence-based approach-the ALBI grade. J Clin Oncol. 2015;33 (6):550-558. doi:10.1200/JCO.2014.57.9151

18. Wang J, Zhang Z, Yan X, et al. Albumin-Bilirubin (ALBI) as an accurate and simple prognostic score for chronic hepatitis B-related liver cirrhosis. Dig Liver Dis. 2019;51(8):1172-1178. doi:10.1016/j. dld.2019.01.011

19. Wai CT, Greenson JK, Fontana RJ, et al. A simple noninvasive index can predict both significant fibrosis and cirrhosis in patients with chronic hepatitis C. Hepatology. 2003;38(2):518-526. doi:10.1053/ jhep.2003.50346

20. Sterling RK, Lissen E, Clumeck N, et al. Development of a simple noninvasive index to predict significant fibrosis in patients with $\mathrm{HIV} / \mathrm{HCV}$ coinfection. Hepatology. 2006;43(6):1317-1325. doi:10.1002/hep.21178

21. Wiesner R, Edwards E, Freeman R, et al. Model for end-stage liver disease (MELD) and allocation of donor livers. Gastroenterology. 2003;124(1):91-96. doi:10.1053/gast.2003.50016

22. DeLong ER, DeLong DM, Clarke-Pearson DL. Comparing the areas under two or more correlated receiver operating characteristic curves: a nonparametric approach. Biometrics. 1988;44(3):837-845. doi: $10.2307 / 2531595$

23. Yu JH, Suh YJ, Jin YJ, et al. Prediction model for hepatocellular carcinoma risk in treatment-naive chronic hepatitis B patients receiving entecavir/tenofovir. Eur $J$ Gastroenterol Hepatol. 2019;31 (7):865-872. doi:10.1097/MEG.0000000000001357

24. Wong VW, Chan SL, Mo F, et al. Clinical scoring system to predict hepatocellular carcinoma in chronic hepatitis B carriers. J Clin Oncol. 2010;28(10):1660-1665. doi:10.1200/JCO.2009.26.2675

25. Kim JH, Kim YD, Lee M, et al. Modified PAGE-B score predicts the risk of hepatocellular carcinoma in Asians with chronic hepatitis B on antiviral therapy. $J$ Hepatol. 2018;69(5):1066-1073. doi:10.1016/j. jhep.2018.07.018

26. Kim C, Um S, Seo Y, et al. Prognosis of hepatitis B-related liver cirrhosis in the era of oral nucleos(t)ide analog antiviral agents. $J$ Gastroenterol Hepatol. 2012;27(10):1589-1595. doi:10.1111/ j.1440-1746.2012.07167.x

27. Wang HW, Lai HC, Hu TH, et al. On-treatment changes in FIB-4 and 1-year FIB-4 values help identify patients with chronic hepatitis $\mathrm{B}$ receiving entecavir therapy who have the lowest risk of hepatocellular carcinoma. Cancers. 2020;12(5). doi:10.3390/cancers12051177 
28. Chen RC, Cai YJ, Wu JM, et al. Usefulness of albumin-bilirubin grade for evaluation of long-term prognosis for hepatitis B-related cirrhosis. J Viral Hepat. 2017;24(3):238-245. doi:10.1111/jvh.12638

29. Liaw YF, Sung JJ, Chow WC, et al. Lamivudine for patients with chronic hepatitis B and advanced liver disease. $N$ Engl J Med. 2004;351(15):1521-1531. doi:10.1056/NEJMoa033364

30. Chen CJ, Yang HI, Su J, et al. Risk of hepatocellular carcinoma across a biological gradient of serum hepatitis B virus DNA level. JAMA. 2006;295(1):65-73. doi:10.1001/jama.295.1.65
31. Zoutendijk R, Reijnders JG, Zoulim F, et al. Virological response to entecavir is associated with a better clinical outcome in chronic hepatitis B patients with cirrhosis. Gut. 2013;62(5):760-765. doi:10.1136/gutjnl-2012-302024

32. Sun Y, Wu X, Zhou J, et al. Persistent low level of hepatitis B virus promotes fibrosis progression during therapy. Clin Gastroenterol Hepatol. 2020;18(11):2582-2591. doi:10.1016/j.cgh.2020.03.001

\section{Publish your work in this journal}

Infection and Drug Resistance is an international, peer-reviewed openaccess journal that focuses on the optimal treatment of infection (bacterial, fungal and viral) and the development and institution of preventive strategies to minimize the development and spread of resistance. The journal is specifically concerned with the epidemiology of antibiotic resistance and the mechanisms of resistance development and diffusion in both hospitals and the community. The manuscript management system is completely online and includes a very quick and fair peerreview system, which is all easy to use. Visit http://www.dovepress.com/ testimonials.php to read real quotes from published authors. 